\title{
Plyometric Performance Assessment of Basketball \& Volleyball Female Players
}

\author{
Enkeleida Lleshi \\ PhD, Sports University of Tirana, Institute of Sport Research \\ Department of Research in Applied Movement
}

\section{Abstract}

Improving the ability to jump is a major training goal for many sports, and drop jump is the well-known training method used to achieve this improvement. The players in team games should be thrown higher than his or her opponent, and this jump should be executed faster than the opponent's jump. Volleyball \& Basketball from the specificity of their training, there are differences in the physical qualities of individual and team sports performance, where one of the elements is vertical jumping in training the plyometric. This study is concentrated on two teams: Women's Volleyball Team (V) and Women's Basketball Team (B) 15 members each, focusing on 30 subjects. Players were measured in physical parameters; Age (V-24: B-27), Body Height (V-180.4cm; B-173.4cm), Body Weight (V-70.37kg; B-63.88kg), BMI (V-21.67\%; B-20.93\%). The players performed the test DJ $40 \mathrm{~cm}$ and DJ60 cm in the platform Leonardo ${ }^{\circledR}$ Ground Force Reaction Plate (GRFP) which expresses Force max (kN), Power max (w/kg), Time Contact (TCs), Air Time (TAs), TA/TCs. Results obtained by GRFP showed different team values of the two sports in the parameters of test from DJ40 $\mathrm{cm}$ and DJ $60 \mathrm{~cm}$. The TA/TC show V1.89>B1.85 in DJ40 test and TA/TC in DJ60 V1.96>B1.71. The results showed statistically significant improvement with respect to $\mathrm{F}$ max $(\mathrm{kN}), \mathrm{F} \max (\mathrm{kg} \mathrm{N})$ and power $(\mathrm{kg} \mathrm{W})$ at the level of $\mathrm{p}=0.05$. Conclusion; The methodology used helps us to compare high and poor performances, which help trainers to program a more detailed plyometric training for the development of athletes' jumping ability. Through the Drop Jump test it can be achieved to be evaluated not only by the height of the development of vertical jump but also by the phase of stay in the air. Through value comparisons, between tests and sports, trainers can develop programmed plyometric.

Keywords: volleyball, drop jump $40 \mathrm{~cm}-60 \mathrm{~cm}$, basketball, plyometric. 


\section{Introduction}

Plyometric training (1) is widely used to improve jumping ability, especially in sports such as volleyball and basketball $(2,3,4,5)$. Volleyboll (V) and Basketboll (B) sports have different performances for their own game characteristic, so the training is specific where there are differences in the physical qualities of individual and team performance. Volleyball players develop tactical and technical elements such as; Attack, Blocking, Service, etc., while basketball players in addition to running on the field and technical elements must jump during penetrations in the area, catching the ball on the board or realizing the shot. One of the main elements of these sports is vertical jumping. By vertical jump we mean the athlete's ability to raise the center of gravity of his body with the help of dynamic work of the muscles of the lower extremities. So the meaning of the vertical jump element is basically the measurement of the strength, strength, speed of the lower extremities. Numerous scholars have found that high jumps can be improved through applications of plyometric exercises. The best possible perfection of a vertical jump is achieved with training with the purpose of increasing the height of the jump, the high degree of muscular activity that is achieved by increasing the loads in the phase of extension of the vertical jump. Drop Jump is a popular form of plyometric training as well as better interpretation of the term plyometry.Plyometric training (6) is widely used to improve the ability to dance, especially in collective sports such as volleyball, basketball, football, etc (7). Many authors show that explosive power is characteristic of professional basketball players not only in volleyball (8). Evaluation of vertical jump through the Drop Jump $40 \mathrm{~cm}$ $60 \mathrm{~cm}$ pryometric test will enable us to compare the values obtained from the strength platform between these sports.

\section{Methodology}

This study is concentrated on two teams: Women's Volleyball Team (V) and Women's Basketball Team (B) 15 members each, focusing on 30 subjects. Players were measured in physical parameters;

Table1 Anthropometric Measurements (Volleyball\&Basketball players)

\begin{tabular}{|c|c|c|c|c|c|}
\hline Team & $\mathrm{Nr}$ & Age & $\begin{array}{c}\mathrm{BH} \\
(\mathrm{cm})\end{array}$ & $\mathrm{BW}(\mathrm{kg})$ & $\begin{array}{c}\mathrm{BMI}( \\
\left.\mathrm{kg} / \mathrm{m}^{2}\right)\end{array}$ \\
\hline Volleyball & 15 & 24 & 180.4 & 70.37 & $21.67 \%$ \\
\hline Basketball & 15 & 27 & 173.4 & 63.88 & $20.93 \%$ \\
\hline
\end{tabular}

\subsection{Protocols of the Test Performed}

After were conducted anthropometric measurements and later on the tests in vertical jump performance of the protocol tests.The players performed the test DJ40cm and DJ60 cm in the system platform Leonardo ${ }^{\circledR}$ Ground Force Reaction Plate (GRFP) which expresses Force max (kN), Power max (w/kg), Time Contact (TCs), Air Time 
(TAs), TA/TCs in University of Sports, Tirana. The players one by one performed on the cube height $40 \mathrm{~cm}$ and $60 \mathrm{~cm}$, with their hands on their loins. Through a free fall from the height of the cube they leave themselves falling into GRFP platform and rapidly the reaction after contacting with GRFP they should jump in vertical as high as possible. The test has been developed 3 times and we got the best measure of the contact time and the time in the air.

\section{Drop Jump Test}

Table2. Data obtained from GRFP (Volleyball\&Basketball players)

\begin{tabular}{|c|c|c|c|c|c|c|c|c|c|c|}
\hline Test & \multicolumn{2}{|c|}{$\begin{array}{l}\text { Fmax } \\
\mathrm{N} / \mathrm{kg}\end{array}$} & \multicolumn{2}{|c|}{$\begin{array}{l}\text { Pmax } \\
\mathrm{w} / \mathrm{kg}\end{array}$} & \multicolumn{2}{|c|}{$\begin{array}{c}\text { Time Contact } \\
\text { TCs } \\
\end{array}$} & \multicolumn{2}{|c|}{$\begin{array}{l}\text { Air Time } \\
\text { TAs }\end{array}$} & \multicolumn{2}{|c|}{ TA/TC } \\
\hline $\begin{array}{l}\text { V15- } \\
\text { B15 } \\
\end{array}$ & Vboll & Bboll & Vboll & Bboll & Vboll & Bboll & Vboll & $\begin{array}{c}\text { Bbol } \\
1\end{array}$ & $\begin{array}{c}\text { Vbol } \\
1\end{array}$ & $\begin{array}{c}\text { Bbol } \\
1\end{array}$ \\
\hline DJ-40 & $\begin{array}{c}48.1 \\
4\end{array}$ & $\begin{array}{c}55.6 \\
2\end{array}$ & $\begin{array}{c}31.4 \\
4\end{array}$ & $\begin{array}{c}26.4 \\
3\end{array}$ & 0.251 & 0.202 & 0.45 & 0.37 & 1.89 & 1.85 \\
\hline DJ-60 & $\begin{array}{c}65.1 \\
9\end{array}$ & $\begin{array}{c}74.0 \\
7\end{array}$ & $\begin{array}{c}31.2 \\
6\end{array}$ & $\begin{array}{c}23.0 \\
6\end{array}$ & 0.231 & 0.198 & $\begin{array}{c}0.43 \\
6\end{array}$ & 0.34 & 1.96 & 1.71 \\
\hline
\end{tabular}

\subsection{Statistical analyses}

An analysis was done to check whether the test and measurement methods used were valid to identify whether groups of Volleyball players and Basketball players achieved high or poor performance.A unilateral analysis of variance (ANOVA) on data from 30 athletes was performed in order to identify changes in the parameters of the DJ $40 \mathrm{~cm}$ DJ60cm test; Scheffe post hoc analysis was run to show differences between groups. T-tests of paired samples were performed to compare DJ40 $\mathrm{cm}$ - DJ $60 \mathrm{~cm}$ parameters. Moreover, a PCA with a Varimax rotation was executed to examine a possible tendency towards the dependence of temporary force or peak between groups of athletes when executing DJ60 cm test. All statistical procedures were performed using the Package Statistics for Social Sciences 10.0.1 software (SPSS) Inc., Chicago, IL). An alpha level of 0.05 was used.

\section{Results}

The results revealed that the Volleyball players were thrown higher ( $p, 0.001$ ) than the Basketball players. From the data obtained from the platform we see that there are different performances between individual players in both sports, despite the fact that in the team average it turned out that volleyball players are higher in the DJ test. 
The graph below clearly shows the level of values obtained by GFRP of the two tests DJ40-DJ60 in Volleyball \& Basketball players.

Graphic.1: Drop Jump 60cm - 40cm test at measurement.

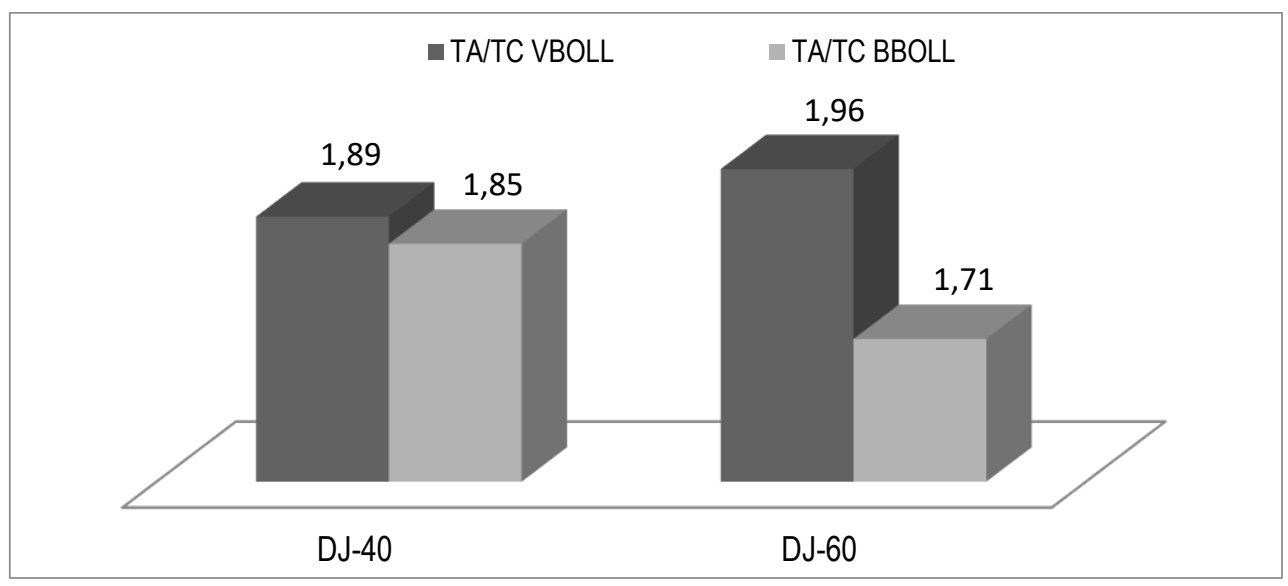

\section{Discussion}

From the data obtained we see that Volleyball players have a better result in time than Basketball players in DJ tests. This test assesses the explosive strength of the lower limbs. The test represents the "Explosive-Reactive-Ballistic Force" and in particular, the neuromuscular capacity to develop very high values of force during the stretchcontraction cycle (9). But the Basketball players showed that in the DJ40cm test they performed better than in the DJ60 cm test. Where on the contrary the volleyball players performed better in DJ60 cm.The DJ $40 \mathrm{~cm}$ and DJ $60 \mathrm{~cm}$ test performance results presented above differ from each other. Strength and power variables were tested for statistically significant differences between the two sports volleyball \& basketball. The aim was to identify any possible changes as to the effects of the training derived from their team training. Analysis of ANOVA with 2 measurements for maximum strength as dependent variable and groups as independent resulted in statistically insignificant values (sig.> 0.05) for both Drop Jump test $40 \mathrm{~cm}$ and Drop Jump test $60 \mathrm{~cm}$. ANOVA analysis with 2 repeated measures for maximum power as a dependent variable and groups as independent resulted in statistically insignificant values (sig.> 0.05 ) for both the DJ $40 \mathrm{~cm}$ test and the DJ $60 \mathrm{~cm}$ test. The linear correlation between the "Drop Jump 40" and "Drop Jump 60" tests as an intuitive assessment would be the connection that exists between the similarity of the test with drop-jump-drop DJ from a height of $40 \mathrm{~cm}$ and that of DJ $60 \mathrm{~cm}$. The correlation between the tests was measured according to the Pearson product moment. The results showed a high correlation coefficient $(r=0.924)$ and statistically significant (sig. <0.0005). The presented results of drop-jump performance in the DJ $40 \mathrm{~cm}$ and DJ60 $\mathrm{cm}$ tests differ from each other but also between the two groups taken in the study to be compared. Strength and power variables were tested for statistically 
significant differences between the two teams. Several factors, including a training program design (the type of exercises, training duration, training frequency, volume and intensity of training), subject characteristics (age, gender, fitness level, sport practice) and methods of testing different types of vertical jumps may be responsible for the conflicting findings concerning plyometric training. Some training studies have found that plyometric training based on DJs does not significantly enhance jump height or lower limb power, while other reports showed a large variability in the magnitude of such enhancement. It has previously been reported (10) an average relationship between strength / measuring power in squat jump performance from place to place and CMJ counter-action jump (movement) in the volleyball team. This platform consists in obtaining more data to the evaluation of the volleyball players, force ( $\mathrm{F}$ max $\mathrm{kN}$ ) and power (P max $\mathrm{kW})$, during the vertical jump.

\section{Conclusion}

Volleyball \& Basketball players despite their plyometric training time over the years, showed a low level of air time flying DJ interpretation. Improving the ability to jump is a major training goal for many sports, and drop jump is the well-known training method used to achieve this improvemen. Individually the players need to improve their jump performance in order to achieve a better personal record. Compared to the reference table of the Reactive Forces Index (1.5-2.0RSI) for the Drop Jump test, we came to the conclusion that the level of Volleyball \& Basketball players in Albania obtained in the result of the TA / TCs Index represents a moderate level of training, at the level of players prepared for moderate plyometric intensity. The values that were reported as high as possible showed that the athletes of both sports did not develop regular plyometric training.

\section{References}

[1] WILT, F. Plyometrics-What it is-How it works. Athl. J.55(76):89-90. 1975.

[2] BLATTNER, S., AND L. NOBLE. Relative effects of isokinetic and plyometric training on vertical jumping performance. Res Q. 50:583-588. 1979.

[3] BROWN, M.E., J.L. MAYHEW, AND M.A. BOLEACH. Effect of plyometric training on vertical jump performance in high school basketball players. J. Sports Med. Phys. Fitness 26:1-4. 1986.

[4] FATOUROS, G.I., Z.A. JAMURTAS, D. LEONTSINI, K. TAXILDARIS, N. AGGELOUSIS, N. KOSTOPOULOS, AND P. BUCKENMEYER. Evaluation of plyometric exercise training, weight training, and their combination on vertical jumping performance and leg strength. J. Strength Cond. Res. 14:470-476. 2000.

[5] HEWETT, T.E., A.L. STROUPE, T.A. NANCE, AND F.R. NOYES. Plyometric training in female athletes. Decreased impact forcforces and increased 
hamstring torques. Am. J. Sports Med. 24: 765-773. 1996

[6] 6.WILT, F. Plyometrics-What it is-How it works. Athl. J. 55(76):89-90. 1975

[7] BOSCO, C., AND P.V. KOMI. (1982). Muscle elasticity in athletes. In: Exercise and Sport Biology. P.V. Komi, R.C. Nelson and C.A. Morehouse, eds. Champaign, IL: Human Kinetics, 1982. pp. 109-117.

[8] 8.Latin, Berg, \& Baechle, 1994; Hoff-man, Tenenbaum, Maresh, \& Kraemer, 1996; Ziv \& Lidor, 2009).

[9] 9. Cometti G., \& Cometti D., 2009. La Pliometria (origini, teorie, allenamento) (2nd ed.) Tivoli: Italy.pg 60-67

[10] 10. Carmelo Bosco,2006. Ph.D."La forza muscolare"-Aspetti Fisiologici ed Applicazioni Pratiche" Capitolo IV, La Forza Esplosiva ,pg103-110. 